\title{
Special volume on 'Recent Developments in Queueing Theory' of the third ECQT conference: part 2
}

\author{
Ivo J. B. F. Adan ${ }^{1} \cdot$ Bernardo D'Auria ${ }^{2}$. Offer Kella ${ }^{3}$ \\ Published online: 26 October 2019 \\ (c) Springer Science+Business Media, LLC, part of Springer Nature 2019
}

The three papers summarized below comprise the second part of the volume dedicated to work selected from the 74 presentations at the third European Conference on Queueing Theory, held in Jerusalem, Israel, July 2-4, 2018. Like the six papers that appeared in the first part (Vol. 93, issues 1-2, 2019), the current three have been chosen, revised and edited for publication through a careful refereeing process. As mentioned in the editorial to the first part, QUESTA also generously published special issues of papers selected from the first and second ECQT meetings (vol. 82, issues 1-2, 2016, vol. 87, issues 3-4, 2017 and vol. 88, issues 1-2, 2018).

The sub-areas to which these papers belong can be categorized as queueinginventory systems, (discrete time) queues with the join-the-shortest-queue discipline and polling models.

Shajin, Krishnamoorthy and Dudin study a queueing-inventory system with reservation of inventory items with the possibility of cancellation and overbooking for future times up to some fixed number of frames. Their model includes a MAP arrival assumption and exponentially distributed service and inter-cancellation times. The duration of one unit of time is approximated by a phase-type distribution. The authors compute system performance characteristics. They further provide a product form solution in the special case of Poisson arrivals and exponentially distributed life times. Numerical examples are given, and optimization problems are investigated.

Offer Kella

offer.kella@gmail.com

Ivo J. B. F. Adan

i.adan@tue.nl

Bernardo D'Auria

bernardo.dauria@uc3m.es

1 Department of Industrial Engineering and Innovation Sciences, Eindhoven University of Technology, P. O. Box 513, 5600 MB Eindhoven, The Netherlands

2 Department of Statistics, Madrid University Carlos III, 28903 Getafe, Madrid, Spain

3 Department of Statistics and Data Science, The Hebrew University of Jerusalem, 9190501 Jerusalem, Israel 
Saxena, Dimitriou, and Kapodistria consider a discrete-time system consisting of two queues fed by a single stream of jobs. Jobs are assigned to each queue according to the join-the-shortest-queue policy. An example of an application for their model is a simple relay-based cooperative wireless network with a single source, two infinite capacity relay nodes, and a common destination with collisions under a load balancing relay scheme. The main idea of the paper is to compute an approximation of the equilibrium distribution of the network by applying a modification of the compensation approach (CA). The challenge of this task lies in the fact that the random walk that describes the system violates a main assumption of the CA technique: that the only transitions allowed are to neighboring states. The authors successfully achieve the extension of the CA methodology to this case and elaborate on an algorithm to compute the approximate equilibrium distribution of the system. They compare their results numerically with the ones obtained by applying the power-series algorithm, the performance of which is inferior.

Van Ommeren, Al Hanbali and Boucherie analyze polling systems with a selfruling service discipline. This means that the server can decide to leave a queue, independent of the queue-length and the number of served customers, or to stay longer even when it is empty. This discipline includes, for example, time-limited and one-limited disciplines, but also a new one referred to as the one-plus-one selfruling discipline. The authors derive a relation between the joint probability generating functions of the number of customers present at the start and at the end of a server visit. This relation is then used to determine the time average transforms of the queue lengths and workload distributions. 